\title{
10. Terapias adyuvantes a la terapia antifibrótica en la fibrosis pulmonar idiopática. Importancia del manejo multidisciplinario
}

\author{
ÁlVARO UNDURRAGA P.*, FELIPE REYES C.**, JOSÉ LUIS VELÁSQUEZ M.***, \\ CLAUDIA AZÓCAR B.****, HERNÁN CABELLO A.*****, MIGUEL AGUAYO C.******, \\ MANUEL VARGAS D.*******, DANIELA MATURANA S.******** y DANIELA DÍAZ H.*********
}

\author{
Adjuvant therapies to antifibrotic therapy in IPF. Importance of multidisciplinary \\ management
}

Idiopathic pulmonary fibrosis (IPF) is characterized by reduced functional capacity, dyspnea and exercise-induced hypoxia, which decreases tolerance to exertion and limits the ability to perform daily activities. Comorbidities are frequent and their presence contribute to worsening quality of life and increased mortality. Therefore, in addition to antifibrotic therapies, patients with IPF benefit of a comprehensive approach to care that may include: screening, diagnosis and treatment of comorbidities, admission to research protocols, symptomatic management, palliative care, supplementary oxygen, pulmonary rehabilitation, education and support by a multidisciplinary team.

Key words: Idiopathic Pulmonary Fibrosis; Comorbidity; Quality of Life; Palliative Care; Oxygen; Patient Care Team.

\section{Resumen}

La fibrosis pulmonar idiopática (FPI) se caracteriza por presentar una capacidad funcional reducida, disnea e hipoxia inducida por el ejercicio, lo que disminuye su tolerancia al esfuerzo y limita su capacidad de realizar actividades diarias. Las comorbilidades son frecuentes y su presencia contribuyen al empeoramiento de la calidad de vida y aumento de la mortalidad. Por lo anterior, es que además de las terapias antifibróticas, los pacientes con FPI se benefician de un enfoque integral de la atención que puede incluir: pesquisa, diagnóstico y tratamiento de comorbilidades, ingreso a protocolos de investigación, manejo sintomático, cuidados paliativos, oxígeno suplementario, rehabilitación pulmonar, educación y apoyo por un equipo multidisciplinario.

Palabras clave: Fibrosis pulmonar idiopática; Comorbidilidad; Calidad de Vida; Cuidado Paliativo; Oxígeno; Equipo de Cuidado de Pacientes.

\footnotetext{
* Profesor Adjunto Universidad de Chile. Instituto Nacional del Tórax y Clínica Las Condes. Santiago, Chile. ** Coordinador Comisión de Enfermedades Pulmonares Intersticiales Difusas, SER Chile. Hospital Clínico Universidad de Chile. Instructor Universidad de Chile. Santiago, Chile.

*** Hospital San Juan de Dios. Clínica Hospital del Profesor. Docente Universidad San Sebastián. Santiago, Chile.

**** Jefe Sección Enfermedades Respiratorias, Departamento de Medicina Interna, Universidad de Concepción. Clínica Universitaria de Concepción. Concepción, Chile.

***** Clínica Alemana de Santiago. Santiago, Chile.

****** Hospital Guillermo Grant Benavente, Concepción. Profesor asistente Universidad de Concepción. Concepción, Chile.

******* Sala ERA: Enfermedades Respiratorias Agudas, Hospital E. Torres G. Iquique, Chile.

$* * * * * * * * \quad$ Hospital Clínico Dra. Eloísa Díaz, La Florida. Santiago, Chile.

$* * * * * * * * *$ Clínica BUPA (British United Provident Association). Santiago, Chile.
} 
La fibrosis pulmonar idiopática (FPI) se caracteriza por presentar una capacidad funcional reducida, disnea e hipoxia inducida por el ejercicio $^{1}$. Las personas con FPI experimentan con frecuencia falta de aire, lo que disminuye su tolerancia al esfuerzo y limita su capacidad de realizar actividades diarias. Además, los pacientes reportan niveles bajos de actividad física y vitalidad y a su vez, altos niveles de disnea y fatiga, lo que determina una peor calidad de vida ${ }^{2}$. Por otro lado, las comorbilidades son comunes, su presencia contribuye al empeoramiento de la calidad de vida y aumento de la mortalidad ${ }^{3}$.

Por lo anterior es que además de las terapias antifibróticas, los pacientes con FPI se benefician de un enfoque integral de la atención que puede incluir: pesquisa, diagnóstico y tratamiento de comorbilidades, ingreso a protocolos de investigación, manejo sintomático, cuidados paliativos, oxígeno suplementario, rehabilitación pulmonar, educación y apoyo por un equipo multidisciplinario.

\section{Comorbilidades}

Como se describe en las guías de la ATS/ ERS/ALAT/JRS 2018, los pacientes con FPI tienen comorbilidades clínicas y subclínicas, la identificación temprana y el tratamiento de las comorbilidades significativas pueden traducirse en una mejoría de la calidad de vida, pronóstico y eventualmente supervivencia.

\section{1) Hipertensión arterial pulmonar (HAP)}

Comorbilidad reportada entre 30 a $86 \%$, según el método empleado para su detección, ecocardiograma o cateterismo cardiaco derecho. Su presencia confiere peor pronóstico, disminución en la capacidad de ejercicio, hipoxemia y aumento de mortalidad.

El ecocardiograma permite estimar la presión sistólica de la arteria pulmonar (PSAP), así como alteraciones estructurales y funcionales del ventrículo derecho. El cateterismo cardiaco se reserva en caso de duda diagnóstica, por lo que se realiza en porcentaje menor de los $\operatorname{casos}^{4}$.

El uso de oxígeno suplementario y el trasplante pulmonar son las terapias para HAP que tienen más evidencia. Se ha explorado el beneficio de sildenafil en estos pacientes en diferentes estudios. El estudio STEP-IPF ${ }^{5}$ no mostró beneficio significativo en desenlace medido de mejoría en $>20 \%$ en distancia en caminata de 6 min (C6M), aunque mejoró mediciones de disnea y calidad de vida en algunos pacientes con disfunción cardíaca derecha (DCD). Recientemente en un análisis de subgrupo del estudio INSTAGE ${ }^{6}$ en pacientes con FPI y $\mathrm{DL}_{\mathrm{CO}}<35 \%$ no se encontró diferencias significativas en los efectos de nintedanib más sildenafil versus nintedanib solo, en los cambios en cuestionario de SGRQ y CVF entre pacientes con o sin signos ecocardiográficos de DCD. Solo se encontraron diferencias en los valores de BNP (brain natriuretic peptide) mayor en los pacientes con $\mathrm{DCD}^{7}$. No existe evidencia actualmente para recomendar una terapia específica en estos pacientes.

\section{2) Combinación de fibrosis pulmonar y enfisema (CFPE)}

El síndrome combinado, tiene relación directa con el consumo de tabaco, $70 \%$ a $80 \%$ de los pacientes con FPI tiene historia de tabaquismo importante. Sin embargo, no todos presentan esta combinación. Se caracteriza en la tomografía computada de alta resolución: TCAR de tórax por la presencia de enfisema en lóbulos superiores y fibrosis pulmonar en lóbulos inferiores. La prevalencia es variable entre el 6 a $67 \%$ en distintas series.

Característicamente las pruebas de función pulmonar muestran CPT y CVF relativamente conservadas, con $\mathrm{DL}_{\mathrm{CO}}$ disminuida desproporcionadamente (más de 1,5 veces).

Se ha observado en este grupo de pacientes, una mayor prevalencia de hipertensión pulmonar y peor pronóstico, por lo que algunos autores lo consideran un fenotipo diferente. Sin embargo, en la actualidad no existen guías diagnósticas o de manejo específico ${ }^{8}$.

\section{3) Cáncer pulmonar}

Los pacientes con FPI tienen 5 veces más riesgo de desarrollar una neoplasia broncogénica pulmonar. Su frecuencia se estima entre un 3 a $22 \%$ mayormente en hombres fumadores y en especial en los portadores de síndrome combinado CFPE. La morbimortalidad es elevada con supervivencia inferior a $30 \%$ el primer año. En un estudio de 103 pacientes con FPI el 20,4\% desarrolló cáncer de pulmón durante un período de observación de hasta 10 años con una incidencia acumulativa creciente con la duración del seguimiento 9 . Los pacientes que reciben tratamiento quirúrgico, quimioterapia y/o radioterapia, tienen mayor morbilidad e incidencia de exacerbaciones agudas. Estudios recientes han sugerido que los antifibróticos previos a la terapia oncológica, pudieran tener un papel en disminuir las complicaciones asociadas al tratamiento ${ }^{10}$. 


\section{4) Trastornos respiratorios del sueño}

Se estima que los trastornos respiratorios del sueño se producen en aproximadamente el $20 \%$ de los adultos mayores y que parece aumentar con la edad. Existe evidencia creciente de la importancia de estos trastornos en patologías pulmonares crónicas. Similar a la enfermedad pulmonar obstructiva crónica (EPOC), en FPI los trastornos respiratorios del sueño podrían tener implicancias en la calidad de vida e incluso en el pronóstico de la enfermedad. Investigaciones han demostrado que el síndrome de apnea e hipopnea obstructiva del sueño (SAHOS) está presente en el $58-88 \%$ de los pacientes con FPI (68\% con SAHOS moderada a grave) $)^{11}$.

La hipoxia nocturna, el SAHOS y el síndrome de piernas inquietas, son los trastornos respiratorios del sueño más frecuentemente reportados. Contrario a lo que podría pensarse, la disminución de los volúmenes pulmonares reduce la estabilidad de las vías respiratorias superiores y aumenta la resistencia de las vías respiratorias altas. Estos cambios pueden facilitar su colapso, especialmente durante el sueño REM, cuando la capacidad residual funcional se reduce aún más debido a la inactividad de los músculos intercostales.

El SAHOS en FPI no solo afecta la calidad de vida o tiene influenza directa en el desarrollo de la HAP, sino que también aumenta el reflujo gastroesofágico y podría acelerar la progresión de la enfermedad debido al estrés oxidativo de la hipoxemia ${ }^{3}$. Además, el SAHOS se relaciona con la morbimortalidad cardiovascular y cerebrovascular.

El tratamiento eficaz con presión positiva continua de la vía aérea (CPAP por sus siglas en inglés) en pacientes con FPI y SAHOS ha mostrado una mejora significativa en la calidad de vida, aunque aún se requieren más estudios para evaluar los posibles beneficios en la progresión y mortalidad de la enfermedad $^{12}$.

\section{5) Comorbilidades cardiovasculares}

La enfermedad coronaria es frecuente, descrita en hasta $25 \%$ de los pacientes con FPI, dada la alta prevalencia en adultos mayores y con antecedentes de tabaquismo. En pacientes sintomáticos está indicado el estudio coronario dirigido. Las calcificaciones en las arterias coronarias como hallazgo en TCAR de tórax, se asocia con ateroesclerosis y daño de la pared del vaso, estos hallazgos pueden ser útiles para establecer un pronóstico de eventos coronarios a futuro ${ }^{13}$.

\section{6) Comorbilidades metabólicas}

Los trastornos metabólicos, especialmente diabetes mellitus son comorbilidades frecuentes. En diferentes series se estima que la prevalencia de diabetes mellitus en pacientes con FPI fluctúa entre un 10 y $39 \%$ en comparación de alrededor del $8 \%$ en la población general ${ }^{14}$. Podría pensarse que el uso de corticoides, terapia estándar hasta el estudio PANTHER, influiría en la alta prevalencia, sin embargo, estos resultados son constantes al excluir a los usuarios de corticoides sistémicos. Aunque los mecanismos causales no son claros, estudios de cohorte poblacional han demostrado que la diabetes mellitus podrían contribuir a la fisiopatología de esta enfermedad y podría considerarse un determinante pronóstico importante de la FPI, sin embargo, el posible impacto del tratamiento y prevención no se conoce.

\section{7) Reflujo gastroesofágico (RGE)}

\section{Recomendaciones, según revisión de la evidencia y metodología GRADE}

¿Se debería realizar tratamiento del RGE en lugar de no hacer nada para el tratamiento de la FPI?

Recomendación 3: La comisión de enfermedades pulmonares difusas de la Sociedad Chilena de Enfermedades Respiratorias sugiere NO utilizar tratamiento farmacológico antirreflujo de rutina en todos los pacientes con fibrosis pulmonar idiopática (recomendación condicional, certeza de la evidencia muy baja)

\section{Consideraciones de subgrupo: Pacientes con reflujo gastroesofágico sintomático y fibrosis pulmonar idiopática podrían beneficiarse del tratamiento}

\section{Resumen de la evidencia}

No se identificaron revisiones sistemáticas (RS) que contestaran esta pregunta, por lo que se realizó una búsqueda de estudios primarios, en la cual se seleccionaron tres estudios observacionales $^{15-17}$. En el (Anexo 1, Apéndice 4), se encuentra la tabla de resumen de hallazgos (SoF) y la tabla de la evidencia a la decisión (EtD).

\section{Beneficios}

El uso de tratamiento antirreflujo en pacientes con fibrosis pulmonar podría disminuir las muertes, la progresión de la enfermedad y podría minimizar la cantidad de personas con disminución absoluta mayor al $10 \%$ en la CVF. 
Por cada 1.000 pacientes que utilizan tratamiento antirreflujo podrían presentar progresión de su enfermedad 45 personas menos $(\mathrm{OR}=0,82$ IC95\% 0,65 a 1,04), disminuir 13 muertes de cada $1.000(\mathrm{OR}=0,8$ IC95\% 0,52 a 1,21$)$ y 31 personas menos podrían presentar disminución absoluta mayor al $10 \%$ en su CVF (OR $=0,76$ IC95\% 0,54 a 1,07).

\section{Riesgos}

El uso de tratamiento antirreflujo en pacientes con fibrosis pulmonar idiopática podría aumentar las infecciones pulmonares. Por cada 1.000 pacientes con fibrosis pulmonar que reciben tratamiento antireflujo de rutina, 30 más podrían tener una infección pulmonar $(\mathrm{OR}=1,91 \mathrm{IC} 95 \%$ 1,04 a 3,52).

\section{Justificación de la recomendación}

El panel consideró que tanto los beneficios como los riesgos del uso de tratamiento antirreflujo en pacientes asintomáticos con fibrosis pulmonar idiopática son pequeños y que el balance entre ambos se inclina a no utilizar el tratamiento.

\section{Consideraciones de implementación}

El uso de la terapia anti RGE, como se explico anteriormente ha mostrado escasos beneficios en disminuir el deterioro de la función pulmonar. Por el contrario, se ha asociado en estudios recientes a aumento de infecciones respiratorias.

En la actualidad no se recomienda el tratamiento anti RGE con inhibidores de bomba de protones (omeprazol, lanzoprazol o esomeprazol) o terapia anti $\mathrm{H}_{2}$ (famotidina y ranitidina) en los pacientes que no presentan síntomas asociados a RGE.

\section{Actualización 2019}

A pesar de que la FPI no está centrada en vía aérea, existe evidencia acumulada respecto a que la aspiración de contenido gástrico puede ser un mecanismo agravante importante en esta enfermedad y otras enfermedades pulmonares intersticiales (EPI) ${ }^{18}$. El reflujo gastroesofágico es frecuente en la FPI (hasta el 90\%) y a menudo es silencioso. En una serie consecutiva de pacientes con FPI, el reflujo confirmado por impedanciometría esofágica-pHmetría $24 \mathrm{~h}$ fue más frecuente en la FPI (en comparación con controles y otras EPI), con correlaciones significativas entre la puntuación de fibrosis en TCAR y el número de eventos de reflujo dista ${ }^{19}$. Además, tanto pepsina como ácidos biliares de muestras de lavado broncoalveolar (LBA) de pacientes con FPI se detectaron comúnmente $\mathrm{y}$ las concentraciones fueron significativamente más altas que las detectadas en pacientes con otras EPI y sujetos de control ${ }^{17}$. Sin embargo, en estos estudios, la bilis se detectó mediante ensayos enzimáticos que no se han validado, específicamente para la medición en líquido del lavado broncoalveolar ${ }^{20}$, por lo tanto, se debe tener precaución al interpretar los resultados.

En las exacerbaciones agudas de FPI (EAFPI), la aspiración gástrica es posiblemente un mecanismo contribuyente. Los patrones de lesión histológica observados en EA-FPI son los de daño alveolar difuso con grados variables de neumonía en organización ${ }^{21}$, que son manifestaciones reconocidas de síndromes de aspiración. Esa aspiración es un precipitante potencial de EA-FPI con el apoyo de una serie de casos y controles, en la que LBA de los pacientes que ingresaron por EA-FPI tenían concentraciones más altas de pepsina en comparación con los controles con FPI estables ${ }^{22}$. Los niveles de pepsina en LBA se correlacionaron con reticulación en TCAR pero no predijeron supervivencia.

Algunas series de estudios observacionales han demostrado un beneficio clínico o espirométrico asociado con la terapia antirreflujo. En una cohorte de 204 pacientes con FPI seguidos en dos centros académicos, el $34 \%$ de los pacientes tenían síntomas de RGE, el $45 \%$ informó antecedentes de RGE y el 47\% informó el uso de medicamentos relacionados con RGE; todos estos factores se asociaron con una mejor supervivencia en el análisis no ajustado ${ }^{23}$. Después del ajuste, la única variable relacionada con RGE asociada con una mejor supervivencia fue el uso de medicamentos para la RGE (razón de riesgo de $0,47, p=0,03$ ), ya sea inhibidores de la bomba de protones o antagonistas $\mathrm{H}_{2}{ }^{23}$. De manera similar, al analizar los sujetos con placebo de tres ensayos clínicos prospectivos realizados por la IPF Clinical Research Network, la terapia antiácida se asoció de forma independiente con una disminución más lenta de la capacidad vital forzada (CVF) a las 30 semanas. Basado en esta evidencia, es que en las Guías ATS/ERS/JRS/ ALAT 2015 de tratamiento de FPI se recomendó condicionalmente (con "muy baja confianza en las estimaciones del efecto") el uso de la terapia antiácida en pacientes con $\mathrm{FPI}^{24}$.

Sin embargo, información posterior de otras cohortes recolectadas prospectivamente no han demostrado consistentemente un beneficio para la terapia antirreflujo, incluso sugieren un posible mayor riesgo de infecciones respiratorias ${ }^{15,25}$. Hay varios estudios clínicos en curso que intentan dilucidar la incógnita. En un ensayo controlado 
aleatorio, doble ciego y en un solo centro, el omeprazol dos veces al día durante 3 meses condujo a una reducción significativa (39\% menor) en la frecuencia de tos medida objetivamente, con aumento no significativo en la frecuencia de infecciones del tracto respiratorio inferior $(26 \%$ vs $14 \%)$ y disminución leve pero preocupante del volumen espiratorio forzado en $1 \mathrm{~s}\left(\mathrm{VEF}_{1}\right)$ y $\mathrm{CVF}^{26}$.

El reconocimiento de que el reflujo gástrico y la microaspiración no se trata solo de la acidez gástrica llevó a un estudio aleatorizado multicéntrico que investigó el papel de la cirugía antirreflujo en pacientes con FPI (CVF $\geq 50 \%)$ con reflujo ácido confirmado objetivamente (puntaje DeMeester $\geq 14,7)^{27}$. Aunque este fue un estudio negativo, las tendencias fueron favorables a las 48 semanas para CVF, mortalidad y exacerbaciones agudas. El estudio también demostró que la cirugía antirreflujo podría realizarse de forma segura en pacientes con FPI cuidadosamente seleccionados ${ }^{27}$.

Hasta que se disponga de más datos, la decisión de tratar el reflujo en pacientes con FPI, ya sea farmacológica o quirúrgica, debe individualizarse y sopesarse cuidadosamente.

\section{8) Depresión}

La depresión y la ansiedad son altamente frecuentes en patologías crónicas y conllevan un aumento en morbilidad. En la Enfermedad Pulmonar Obstructiva Crónica se ha comunicado que entre el 10 y el $60 \%$ están deprimidos y entre el 10 y el $19 \%$ son víctimas de la ansiedad. No sabemos de estadísticas semejantes en FPI, sin embargo, se estima una incidencia creciente 1,5 a 7 veces más probable que en población normal, con un alto impacto en la calidad de vida, por lo que la detección activa de esta comorbilidad es recomendada ${ }^{28}$.

\section{Manejo sintomático}

Es importante priorizar los síntomas y al tratarlos ser específico acerca de las drogas, las dosis, los tiempos y los resultados esperados. Muchos de estos enfermos son de edad avanzada y, por lo tanto, debe ponerse especial atención en las comorbilidades, polifarmacia y en los posibles efectos adversos de los tratamientos.

Hay dos síntomas fundamentales en esta enfermedad que son la tos y la disnea.

\section{1) Tos}

Es universal, pero hay un grupo de pacientes en que la tos es extremadamente molesta y constante. En primer lugar, debe descartarse y/o tratarse otras causas que a menudo coexisten en estos pacientes y que pueden ser responsables de la tos o participar en su origen. Ejemplos de ello son el RGE, la apnea de sueño, la EPOC, el uso de inhibidores de la enzima convertidora de angiotensina, la sinusitis crónica, el síndrome de tos de vía aérea superior, el cáncer pulmonar o la infección respiratoria.

La tos crónica tiene diversos recursos terapéuticos de los cuales podemos echar mano para el manejo. La terapia convencional incluye la codeína especialmente pura (10-20 mg cada 4-6 h, no sobrepasar $120 \mathrm{mg}$ en $24 \mathrm{~h}$, es importante recordar que múltiples jarabes disponibles en el comercio que contienen codeína incluyen también otros componentes que pueden ser perjudiciales en pacientes añosos.

Sus efectos adversos principales son la náusea, los vómitos, el estreñimiento y la tolerancia. Puede ser adictiva. El dextrometorfano es también útil cuando se dispone de él, sin embargo, en Chile actualmente no se encuentra disponible. Ocasionalmente un ensayo con dosis bajas de prednisona suele ser útil para sedar la tos, pero si luego de dos semanas no hay efecto, es preciso suspenderla, debido a los riesgos ya conocidos en el estudio PANTHER ${ }^{29}$.

La talidomida es un potente fármaco inmunomodulador, antiinflamatorio y antiangiogénico, se utiliza actualmente como tratamiento eficaz en el mieloma múltiple, síndromes mielodisplásico entre otros. La talidomida, se ha explorado en el manejo de la tos en pacientes con $\mathrm{FPI}^{30}$ en dosis de 50 a $100 \mathrm{mg} 1 \mathrm{vez}$ al día, con buenos resultados. En la práctica clínica se ha reservado en última instancia, en los pacientes refractarios a tratamientos convencionales dado los efectos adversos importantes como cefalea, somnolencia, bradicardias extremas, disnea brusca, dolor torácico, incluso riesgo protrombótico.

La gabapentina, es otro fármaco empleado en el manejo de la tos, en dosis 300-1.800 mg con menor eficacia que el anterior pero un mejor perfil de seguridad ${ }^{31}$.

La literatura ha publicado algunos antitusígenos que no disponemos como el cromolin sódico en altas dosis nebulizado y un inhibidor de kinasas: el omipalisib (GSK 2126458).

Otro recurso excepcional pero del cual hemos tenido ocasionales buenos resultados es el sulfato de morfina en dosis de 5 a $10 \mathrm{mg}$ cada $12 \mathrm{~h}$.

La terapia anti-fibrótica no ha demostrado cambios en los síntomas en los estudios clínicos. Sin embargo, en los estudios de vida real, la pirfenidona ha demostrado mejoría en la tos ${ }^{32}$. 


\section{2) Disnea}

En etapas tempranas se describe en un $20 \%$, sin embargo, en las etapas avanzadas todos los pacientes la padecen debido al aumento de la demanda ventilatoria que tienen estos pacientes. Al igual que en la tos, otras comorbilidades pueden influir o aumentar la disnea: la debilidad muscular, el aumento de peso, la hipertensión pulmonar, el SAHOS y aún causas psicopatológicas deben ser consideradas como causantes o colaboradores de la disnea.

Hay poca evidencia en la literatura con la terapia farmacológica ${ }^{33}$. Sin embargo, en base a la experiencia clínica hemos tenido muy buenos resultados ocasionales con la morfina que al igual que la tos puede ser un recurso de gran utilidad.

Otro recurso útil como paliativo en las crisis de disnea es el uso de ventiladores manuales colocados a $15 \mathrm{~cm}$ de la nariz. Hay un autor que ha propuesto una curiosa explicación sobre este resultado del chorro de aire en nariz que "engañaría al cerebro" logrando alivio de la disnea ${ }^{34}$.

\section{Oxigenoterapia}

Recomendaciones, según revisión de la evidencia y metodología GRADE.

En pacientes con fibrosis pulmonar idiopática que presenten hipoxemia o hipertensión pulmonar, ¿se debería utilizar oxígeno?

Recomendación 4: En pacientes con fibrosis pulmonar idiopática que presenten hipoxemia (en reposo, ejercicio o durante el sueño) o bien hipertensión pulmonar, la comisión de enfermedades pulmonares difusas sugiere el uso de oxígeno por sobre aire ambiental (recomendación condicional basada en certeza baja de los efectos)

\section{Resumen de la evidencia}

Identificamos una revisión sistemática relevante para la pregunta ${ }^{35}$. Esta revisión identificó tres $^{36-38}$ estudios aleatorizados evaluando el uso de oxígeno versus aire ambiental. En total, en los tres estudios, se incluyó a 98 pacientes con fibrosis pulmonar idiopática y desaturación al ejercicio (Anexo 1, apéndice 5), se encuentra la tabla de resumen de hallazgos (SoF) y la tabla de la evidencia a la decisión (EtD)).

\section{Beneficios}

Reanalizando la información de los estudios identificados en la revisión sistemática, encontramos que el uso suplementario de oxígeno podría llevar a una reducción de la intensidad de disnea de 0,06 unidades medida en la escala de Bohr (IC 95\% 0,24 menor a 0,13 más alto). La escala de Bohr evalúa la disnea con puntajes de 1 a 10 , donde los puntajes más altos se traducen en mayor disnea. La diferencia mínima observada es de 1 punto $^{51}$, lo cual sugiere que la diferencia encontrada de 0,06 unidades es probablemente trivial.

En opinión del panel, los pacientes podrían sentirse aliviados con el uso de oxígeno, lo que podría traer consigo una mejoría de la calidad de vida y una mejor tolerancia al esfuerzo. Sin embargo, esto no ha sido adecuadamente estudiado.

\section{Riesgos \\ No se identificaron riesgos asociados al uso de oxígeno.}

\section{Justificación de la recomendación}

El panel consideró que el uso de oxígeno podría ayudar a disminuir los síntomas del paciente en un contexto en que no hay muchas alternativas efectivas. No obstante, hay incertidumbre sobre la efectividad real del oxígeno en pacientes con fibrosis pulmonar idiopática.

\section{Consideraciones de implementación}

Los programas de oxígeno domiciliario en Chile son gestionados principalmente para EPOC, con indicaciones precisas y demostradas por evidencia solidad. Fibrosis pulmonar no tiene indicación clara ni evidencia especifica que muestre los beneficios. En la práctica clínica habitual, se utiliza oxígeno domiciliario de forma continua en aquellos pacientes que cumplen criterios de hipoxemia crónica y en reposo. Sin embargo, la indicación de oxígeno durante el ejercicio es más controversial y no existe evidencia que lo respalde.

En Chile, ya que la fibrosis pulmonar no se encuentra dentro del grupo de patologías GES, la indicación de oxígeno domiciliario debe ser financiado por el paciente o en algunas ocasiones por el hospital público en el cual se controla el paciente.

\section{Actualización 2019}

La oxigenoterapia por largo tiempo (OTLT) es aquella utilizada durante al menos $15-18 \mathrm{~h}$ por día para pacientes con hipoxemia en reposo. Los estudios NOTT y MRC trial, ambos en pacientes con EPOC y publicados hace más de 30 años, han proporcionado evidencia suficiente del beneficio en supervivencia con OTLT en la EPOC ${ }^{39,40}$. Sin embargo en pacientes con FPI la evidencia no es tan clara: una revisión Cochrane el 2016 con 3 trabajos incluidos con oxigenoterapia ambulatoria 
(OTA) o en cortos pulsos no declaró suficiente información para respaldar ni rechazar su utilidad en pacientes con enfermedades pulmonares intersticiales ${ }^{35}$.

La OTA, implica el uso de un dispositivo portátil de suministro de oxígeno durante el ejercicio o las actividades de la vida diaria. Esta terapia puede indicarse en aquellos pacientes que presentan caída en la $\mathrm{SpO}_{2}$ con el ejercicio a $<80 \%$ durante $\geq 5 \mathrm{~min}$ y $/ \mathrm{o}<90 \%$ durante $\geq 10 \mathrm{~s}^{41}$. Esta terapia se puede recetar como una intervención independiente para pacientes con hipoxemia de esfuerzo aislada o como un suplemento a un concentrador de oxígeno estacionario para OTLT.

En estudios de laboratorio, la oxigenoterapia suplementaria en ejercicio, ha demostrado mejorar el rendimiento del ejercicio durante las pruebas de laboratorio y reducción en la disnea de esfuerzo $^{42}$. Sin embargo en estudios de vida real estos resultados no han logrado reproducirse. El estudio $\mathrm{AmbOx}^{43}$ no mostró cambios clínico significativos, pero si en el cuestionario de calidad de vida de King's Brief (K-BILD).

A pesar de la evidencia clínica, las guías clínicas actuales recomiendan el uso de oxígeno OTLT para pacientes con EPI con hipoxemia significativa en reposo, definida como $\mathrm{PaO}_{2}$ en reposo $\leq 55 \mathrm{mmHg}$ o $56-59 \mathrm{mmHg}$ con evidencia de hipoxemia crónica (cor pulmonar, policitemia e/o hipertensión pulmonar). En contraste, las recomendaciones para OTA varían sustancialmente entre las diferentes guías clínicas, se recomienda considerar el uso de OTA en pacientes con EPI que experimentan disnea o limitación de ejercicio debido a la desaturación de esfuerzo ${ }^{44}$. Parece relevante considerar las desaturación de oxígeno nocturna: valores mayores a un $10 \%$ del tiempo total del sueño con saturación de $\mathrm{O}_{2}<90 \%$ está asociado al desarrollo de enfermedad vascular pulmonar. Estos hallazgos resaltan la necesidad de realizar estudios nocturnos de oxigenoterapia para evaluar los efectos sobre la HAP y la mortalidad de sujetos con FPI con desaturaciones de oxígeno nocturnas ${ }^{45}$. Aunque es necesaria la evaluación caso a caso y la disponibilidad de recursos, dado la escases de evidencia clínica en esta área.

\section{Rehabilitación respiratoria}

\section{Recomendaciones, según revisión de la evidencia y metodología GRADE}

En pacientes con fibrosis pulmonar idiopática, ¿debería usarse rehabilitación pulmonar vs manejo convencional sin rehabilitación?
Recomendación 5: En pacientes con fibrosis pulmonar idiopática, la comisión de enfermedades pulmonares difusas sugiere realizar rehabilitación pulmonar en vez de manejo convencional sin rehabilitación (recomendación condicional basada en certeza baja de los efectos)

\section{Resumen de la evidencia}

No identificamos ninguna revisión sistemática relevante a la pregunta, por lo que se hizo una búsqueda de estudios primarios. En ella identificamos 4 estudios aleatorizados ${ }^{46-49}$, con evaluación de 3 a 12 semanas de ejercicios supervisados en pacientes con fibrosis pulmonar idiopática (Anexo 1, apéndice 5), se encuentra la tabla de resumen de hallazgos (SoF) y la tabla de la evidencia a la decisión (EtD)).

\section{Beneficios}

Ninguno de los estudios identificados reportó el efecto de la rehabilitación pulmonar en la mortalidad o en la frecuencia de complicaciones. No obstante, es probable que la rehabilitación pulmonar reduzca los síntomas globales medidos mediante el St George's Respiratory Questionnaire (SGRQ) (MD 8,1 menor, IC 95\% 10,7 a 5,4 menos). Este instrumento evalúa síntomas en una escala de 1 a 100, donde los puntajes más bajos representan una menor sintomatología. Diferencias de 4 puntos son habitualmente consideradas importantes para los pacientes.

Además, la rehabilitación pulmonar probablemente aumenta la tolerancia al ejercicio, la que es evaluada mediante el test de caminata en seis minutos. Pacientes sometidos a rehabilitación logran en promedio 47,9 metros más que quienes no han tenido rehabilitación (IC 95\% 26,8 a 69 metros más). Una diferencia de 27 metros es considerada un cambio importante en este test.

\section{Riesgos}

No se identificaron riesgos producto de la rehabilitación pulmonar.

\section{Justificación de la recomendación}

El panel consideró que los beneficios de la rehabilitación pulmonar superan a los potenciales riesgos, costos o incomodidades para el paciente. Sin embargo, en la actualidad constituye una intervención difícil de implementar dada la escasez de centros que la ofrecen.

\section{Consideraciones de implementación}

La rehabilitación pulmonar es la intervención no farmacológica más efectiva. Utilizada para 
aumentar la capacidad de ejercicio, reducir la disnea y mejorar la calidad de vida.

Su implementación se encuentra a cargo principalmente del equipo de kinesiología. Si bien la intervención es de bajo costo, la implementación de este requiere la adquisición de equipos específicos y la capacitación del personal de salud para asegurar la seguridad de pacientes con grave deterioro del intercambio gaseoso, durante el ejercicio.

\section{Actualización 2019}

La rehabilitación pulmonar es la intervención no farmacológica más efectiva. Utilizada para aumentar la capacidad de ejercicio, reducir la disnea y mejorar la calidad de vida. Los kinesiólogos son los profesionales fundamentales en el plan terapéutico de los pacientes con FPI. La enfermedad tiene aumento de limitaciones fisiológicas por su condición lo cual disminuye su capacidad de ejercicio, aumentan su síntomas y disminuye su calidad de vida. Un buen programa de ejercicios mejora la fisiología cardiovascular, pulmonar y de los músculos esqueléticos, mejora la calidad de vida y la capacidad de ejercicio, disminuye los síntomas y mejora por lo tanto el estado de salud.

La rehabilitación pulmonar (RP) mejora la capacidad funcional evaluada mediante el test de marcha y reduce la disnea (escala de Borg) ( $^{50,51}$. La RP implica acondicionamiento aeróbico, entrenamiento de fuerza y flexibilidad, charlas educativas $\mathrm{y}$ intervenciones nutricionales ${ }^{52,53}$. Además, se sabe que la RP tiene beneficios psicosociales positivos que ayudan a los pacientes a entender su enfermedad y mitigar la ansiedad y la depresión. En el Anexo 3, se hace mayor hincapié sobre la importancia de la rehabilitación pulmonar y los desafíos en la implementación de este.

\section{Cuidados paliativos y manejo del fin de la vida}

El objetivo de los cuidados paliativos es mejorar la calidad de vida y cuidar un buen morir de pacientes con FPI, junto a sus familiares y cuidadores. Incluye manejo de síntomas, soporte psicológico y social, rehabilitación y planificación del fin de la vida. Los cuidados paliativos deberían ser ofrecidos en todas las etapas de la enfermedad. Idealmente los hospitales deberían contar con grupos de soporte para pacientes con insuficiencia respiratoria terminal, ser integrados al programa de cuidados paliativos general para evaluar soporte al final de la vida (opioides, oxígeno, ventilación no invasiva etc. $)^{54}$.

\section{¿Dónde fallece el paciente con FPI?}

Si consideramos que estamos tratando con una enfermedad incurable, de peor pronóstico que muchos cánceres, esta pregunta que muy raramente nos formulamos, adquiere plena vigencia. La revista The Economist en uno de sus números recientes, publica las respuesta a grupos de entrevistados de Estados Unidos, Italia, Japón y Brasil ofreciendo como alternativas el hospital, casas de ancianos o el propio hogar. Entre esas alternativas una gran mayoría opta por la preferencia de morir en su hogar ${ }^{55}$.

Sin embargo, otra publicación que investigó dónde mueren efectivamente los pacientes con FPI nos revela que casi el $80 \%$ de los pacientes efectivamente fallecen en el hospital y sólo un $8 \%$ fallecen en su casa ${ }^{56}$. La publicación del Economist aludida revela que no más de un $10 \%$ de los pacientes declaran haber hablado el tema con sus tratantes. Es preocupante también que investigados los tiempos en que se dan las órdenes de "no resucitar" o las "decisiones de fin de vida" la gran mayoría se hacen en los últimos días de vida de los pacientes.

\section{La importancia de una intervención multidisciplinaria}

El concurso de la enfermera, técnicos de enfermería de nivel superior y otros profesionales no médicos es de extraordinaria utilidad: reforzar las indicaciones, explicaciones complementarias, detallar la forma de administración de los medicamentos y sus cuidados, aclarar procedimientos como broncofibroscopía, biopsias por videotoracoscopia, tranquilizan al paciente. Además, las enfermeras con su proximidad con los enfermos pueden hacerlos partícipes en la terapia. Es importante también la colaboración de la enfermera en asegurar el control regular de estos pacientes que será de gran importancia para evaluar la historia de la enfermedad en cada caso ya sea con o sin tratamiento. Existen estudios que han evaluado los sentimientos y percepciones de pacientes con FPI, lográndose identificar tres fases emocionales expresadas al momento del diagnóstico, aceptación y propiedad de la condición. Cada fase requiere de distintos tipos de soporte emocional $^{57}$.

Además, se identificaron cuatro áreas que preocupan al paciente: aspecto físico, soporte familiar, interacción con el mundo médico y esperanza en los avances científicos. Los síntomas depresivos, también son frecuentes en los pacientes con enfermedad pulmonar difusa, lo que lleva a empeoramiento de disnea y calidad del sueño. 
Existen varias formas en las cuales el soporte educacional y psicológico puede ser entregado a paciente con FPI:

1. Formación de grupos de pacientes con FPI.

2. Conferencias grupales, es importante que los clínicos proporcionen información actualizada.

3. La terapia cognitiva-conductual es eficaz en el manejo de pacientes EPOC y puede ser una técnica a utilizar en pacientes con FPI y la intervención farmacológica para la ansiedad y la depresión puede tener un papel fundamental.

\section{Bibliografía}

1.- DOWMAN L, HILL CJ, HOLLAND AE. Pulmonary rehabilitation for interstitial lung disease.. Cochrane Database of Systematic Reviews 2014; 6: CD006322.

2.- CHANG, JA, CURTIS JR, PATRICK DL, RAGHU G. Assessment of health-related quality of life in patients with interstitial lung disease. Chest 1999; 116: 1175-82.

3.- RAGHU G, AMATTO VC, BEHR J, STOWASSER S. Comorbidities in idiopathic pulmonary fibrosis patients: a systematic literature review. Eur Respir J 2015; 46: 1113-30.

4.- OLDHAM J, COLLARD H. Comorbid conditions in Idiopathic Pulmonary Fibrosis: recognition and management. Front Med (Lausanne) 2017; 4: 123.

5.- ZISMAN DA, SCHWARZ M, ANSTROM KJ, COLLARD HR, FLAHERY HR, et al; Idiopathic Pulmonary Fibrosis Clinical Research Network. A controlled trial of sildenafil in advanced idiopathic pulmonary fibrosis. N Engl J Med 2010; 363: 620-8.

6.- KOLB M, RAGHU G, WELLS AU, BEHR J, RICHELDI L, SCHINZEL B, et al. Nintedanib plus sildenafil in patients with idiopathic pulmonary fibrosis. N Engl J Med 2018; 379: 1722-31.

7.- BEHR J, KOLB M, SONG JW, LUPPI F, SCHINZEL B, STOWASSER S, et al. Nintedanib and sildenafil in patients with idiopathic pulmonary fibrosis and right heart dysfunction (INSTAGE): a pre-specified subgroup analysis of a double-blind, randomized clinical trial. Am J Resp Crit Care Med 2019; Jul 31: doi: 10.1164/rccm.201903-0488OC.

8.- PAPAIOANNOU AI, KOSTIKAS K, MANALI ED, PAPADAKI G, ROUSSOU A, KOLILEKAS L, et al. Combined pulmonary fibrosis and emphysema: The many aspects of a cohabitation contract. Respir Med 2016; 117: 14-26.

9.- OZAWA Y, SUDA T, NAITO T, ENOMOTO N, HASHIMOTO D, FUJISAWA T, et al. Cumulative incidence of and predictive factors for lung cancer in IPF. Respirology 2009; 14: 723-8.

10.- IWATA T, YOSHIDA S, FUJIWARA T, WADA H, NAKAJIMA T, SUZUKI H, et al. Yoshino I2. Effect of Perioperative Pirfenidone Treatment in Lung Cancer Patients With Idiopathic Pulmonary Fibrosis. Ann Thorac Surg 2016; 102: 1905-10.

11.- GILLE T, DIDIER M, BOUBAYA M, MOYA L, SUTTON A, CARTON Z, et al. Obstructive sleep apnoea and related comorbidities in incident idiopathic pulmonary fibrosis. Eur Respir J 2017;49. doi:10.1183/13993003.

12.- MERMIGKIS C, BOULOUKAKI I, ANTONIOU K, PAPADOGIANNIS G, GIANNARAKIS I, VOARUCHAKIS G, et al. Obstructive sleep apnea should be treated in patients with idiopathic pulmonary fibrosis. Sleep Breath 2015; 19: 385-91.

13.- AGRAWAL A, VERMA I, SHAH V, AGARWAL A, SIKACHI RR. Cardiac manifestations of idiopathic pulmonary fibrosis. Intractable Rare Dis Res 2016; 5: 70-5.

14.- HYLDGAARD C, HILBERG O, BENDSTRUP E. How does comorbidity influence survival in idiopathic pulmonary fibrosis? Respir Med 2014; 108: 647-53.

15.- KREUTER M, WUYTS W, RENZONI E, KOSCHEL D, MAHER TM, KOLB M, et al. Antacid therapy and disease outcomes in idiopathic pulmonary fibrosis: A pooled analysis. Lancet Respir Med 2016; 4: 381-9.

16.- KREUTER M, SPAGNOLO P, WUYTS W, RENZONI E, KOSCHEL D, BONELLA F, et al. Antacid Therapy and Disease Progression in Patients with Idiopathic Pulmonary Fibrosis Who Received Pirfenidone. Respiration 2017; 93 (6): 415-23.

17.- LEE JS, COLLARD HR, ANSTROM KJ, MARTINEZ FJ, NOTH I, ROBERTS RS, et al. Anti-acid treatment and disease progression in idiopathic pulmonary fibrosis: an analysis of data from three randomised controlled trials. Lancet Respir Med 2013; 1: 369-76.

18.- TOBIN RW, POPE CE 2ND, PELLEGRINI CA, EMOND MJ, SILLERY J, RAGHU G. Increased prevalence of gastroesophageal reflux in patients with idiopathic pulmonary fibrosis. Am J Respir Crit Care Med 1998; 158: 1804-8.

19.- SAVARINO E, CARBONE R, MARABOTTO E, FURNARI M, SCONFIENZA L, GHIO M, et al. Gastro-oesophageal reflux and gastric aspiration in idiopathic pulmonary fibrosis patients. Eur Respir J 2013; 42: 1322-31.

20.- PARIKH S, BROWNLEE IA, ROBERTSON AG, MANNING NT, JOHNSON GE, BRODLIE M, et al. Are the enzymatic methods currently being used to measure bronchoalveolar lavage bile salt levels fit for purpose? J Heart Lung Transplant 2013; 32: 418-23.

21.- RYERSON CJ, COTTIN V, BROWN KK, COLLARD HR. Acute exacerbation of idiopathic pulmonary fibrosis: shifting the paradigm. Eur Respir J 2015; 46: 512-20.

22.- LEE JS, SONG JW, WOLTERS PJ, ELICKER BM, KING TE JR, KIM DS, et al. Bronchoalveolar lavage 
pepsin in acute exacerbation of idiopathic pulmonary fibrosis. Eur Respir J 2012; 39: 352-8.

23.- LEE JS, RYU JH, ELICKER BM, LYDELL CP, JONES KD, WOLTERS PJ, et al. Gastroesophageal reflux therapy is associated with longer survival in patients with idiopathic pulmonary fibrosis. Am J Respir Crit Care Med 2011; 184: 1390-4.

24.- RAGHU G, ROCHWERG B, ZHANG Y, GARCIA CA, AZUMA A, BEHR J, et al. An Official ATS/ERS/ JRS/ALAT Clinical Practice Guideline: Treatment of Idiopathic Pulmonary Fibrosis. An Update of the 2011 Clinical Practice Guideline. Am J Respir Crit Care Med 2015; 192: e3-19.

25.- JO HE, CORTE TJ, GLASPOLE I, GRAINGE C, HOPKINS PMA, MOODLEY Y, et al. Gastroesophageal reflux and antacid therapy in IPF: analysis from the Australia IPF Registry. BMC Pulm Med 2019; 19: 84.

26.- DUTTA P, FUNSTON W, MOSSOP H, RYAN V, JONES R, FORBES R, et al. Randomised, doubleblind, placebo-controlled pilot trial of omeprazole in idiopathic pulmonary fibrosis. Thorax 2019; 74: 346-53.

27.- RAGHU G, PELLEGRINI CA, YOW E, FLAHERTY KR, MEYER K, NOTH I, et al. Laparoscopic antireflux surgery for the treatment of idiopathic pulmonary fibrosis (WRAP-IPF): a multicentre, randomised, controlled phase 2 trial. Lancet Respir Med 2018; 6: 707-14.

28.- LEE JL, CHOI SM, LEE YJ, CHO Y-J, YOON HI, LEE JH, et al. Clinical impact of depression and anxiety in patients with idiopathic pulmonary fibrosis. PLoS One 2017; 12: e0184300.

29.- RAGHU G, ANSTROM KJ, KING TE JR, LASKY JA, MARTÍNEZ FJ, et al; Idiopathic Pulmonary Fibrosis Clinical Research Network. Prednisone, azathioprine, and $\mathrm{N}$-acetylcysteine for pulmonary fibrosis. N Engl J Med 2012; 366: 1968-77.

30.- HORTON MR, SANTOPIETRO V, MATHEW L, HORTON KM, POLITO AJ, LIU MC, et al. Thalidomide for the treatment of cough in idiopathic pulmonary fibrosis: a randomized trial. Ann Intern Med 2012; 157 : 398-406.

31.- SHI G, SHEN Q, ZHANG C, MA J, MOHAMMED $\mathrm{A}, \mathrm{ZHAO} \mathrm{H}$. Efficacy and safety of gabapentin in the treatment of chronic cough: a systematic review. Tuberc Respir Dis (Seoul) 2018; 18: 167-74.

32.- VAN MANEN MJG, BIRRING SS, VANCHERI C, VINDIGNI V, RENZONI E, RUSSELL AM, et al. Effect of pirfenidone on cough in patients with idiopathic pulmonary fibrosis. Eur Respir J 2017; 50.pii: 1701157.

33.- KOHBERG C, ANDERSEN CU, BENDSTRUP E. Opioids: an unexplored option for treatment of dyspnea in IPF. Eur Clin Respir J 2016; 3: 10.3402/ecrj. v3.30629.

34.- GALBRAITH S, FAGAN P, PERKINS P, LYNCH A, BOOTH S. Does the use of a handheld fan improve chronic dyspnea? A randomized, controlled, crossover trial. J Pain Symptom Manage 2010; 39: 831-8.

35.- SHARP C, ADAMALI H, MILLAR AB. Ambulatory and short-burst oxygen for interstitial lung disease. Cochrane Database Syst Rev 2016; 7: CD011716.

36.- TROY L, YOUNG I, MUNOZ P, TAYLOR N, WEBSTER S, LAU E, et al. Does supplemental oxygen increase exercise endurance in patients with idiopathic pulmonary fibrosis? Respirology 2014; 19: 95.

37.- NISHIYAMA O, MIYAJIMA H, FUKAI Y, YAMAZAKI R, SATOH R, YAMAGATA T, et al. Y. Effect of ambulatory oxygen on exertional dyspnea in IPF patients without resting hypoxemia. Respir Med 2013; 107: 1241-6.

38.- ARIZONO S, TANIGUCHI H, SAKAMOTO K, KONDOH Y, KIMURA T, KATAOKA K, et al. Benefits of supplemental oxygen on exercise capacity in IPF patients with exercise-induced hypoxemia. Eur Respir J 2015; 46, S59: OA4971.

39.- NOCTURNAL OXYGEN THERAPY TRIAL GROUP. Continuous or nocturnal oxygen therapy in hypoxemic chronic obstructive lung disease: a clinical trial. Ann Intern Med 1980; 93: 391-8.

40.- MEDICAL RESEARCH COUNCIL WORKING PARTY. Long term domiciliary oxygen therapy in chronic hypoxic cor pulmonale complicating chronic bronchitis and emphysema. Report of the Medical Research Council Working Party. Lancet 1981; 1: 681-6.

41.- LONG-TERM OXYGEN TREATMENT TRIAL RESEARCH GROUP. A randomized trial of long-term oxygen for COPD with moderate desaturation. N Engl J Med 2016; 375: 1617-27.

42.- BELL EC, COX NS, GOH N, GLASPOLE I, WESTALL GP, WATSON A, et al. Oxygen therapy for interstitial lung disease: a systematic review. Eur Respir Rev 2017; 26: 160080.

43.- VISCA D, MORI L, TSIPOURI V, FLEMING S, FIROUZI A, BONINI M, et al. Effect of ambulatory oxygen on quality of life for patients with fibrotic lung disease (AmbOx): a prospective, open-label, mixedmethod, crossover randomised controlled trial. Lancet Respir Med 2018; 6: 759-70.

44.- KHOR YH, RENZONI EA, VISCA D, MCDONALD $\mathrm{CF}$, GOH NSL. Oxygen therapy in COPD and interstitial lung disease: navigating the knowns and unknowns. ERJ Open Res 2019; 5: 00118-2019.

45.- TROY LK, YOUNG IH, LAU EMT, WONG KKH, YEE BJ, TORZILLO PJ, et al. Nocturnal hypoxaemia is associated with adverse outcomes in interstitial lung disease. Respirology 2019; 24: 996-1004.

46.- NISHIYAMA O, KONDOH Y, KIMURA T, KATO K, KATAOKA K, OGAWA T, et al. Effects of pulmonary rehabilitation in patients with idiopathic pulmonary fibrosis. Respirology 2008; 13: 394-9.

47.- DOWMAN LM, MCDONALD CF, HILL CJ, LEE AL, BARKER K, BOOTE C, et al. The evidence of 
benefits of exercise training in interstitial lung disease: a randomised controlled trial. Thorax 2017; 72: $610-9$.

48.- VAINSHELBOIM B, KRAMER MR, FOX BD, IZHAKIAN S, SAGIE A, OLIVEIRA J. Supervised exercise training improves exercise cardiovascular function in idiopathic pulmonary fibrosis. Eur J Phys Rehabil Med 2017;53:209-18.

49.- JACKSON RM, GÓMEZ-MARÍN OW, RAMOS CF, SOL CM, COHEN MI, GAUNAURD IA, et al. Exercise limitation in IPF patients: a randomized trial of pulmonary rehabilitation. Lung 2014; 192: 367-76.

50.- FERREIRA A, GARVEY C, CONNORS GL, HILLING L, RIGLER J, FARRELL S, et al. Pulmonary rehabilitation in interstitial lung disease: benefits and predictors of response. Chest 2009; 135: 442-7.

51.- BOLTON CE, BEVAN-SMITH EF, BLAKEY JD, CROWE P, ELKIN SL, GARROD R, et al. British Thoracic Society guideline on pulmonary rehabilitation in adults 2013; Vol. 68 Suppl 2: ii1-ii30.

52.- ROCHESTER CL, VOGIATZIS I, HOLLAND AE, LAREAU SC, MARCINIUK DD, PUHAN MA, et al. An Official American Thoracic Society/European Respi- ratory Society Policy Statement: Enhancing Implementation, Use, and Delivery of Pulmonary Rehabilitation. Am J Respir Crit Care Med 2015; 192: 1373-86.

53.- DOWMAN LM, MCDONALD CF, HILL CJ, LEE AL, BARKER K, BOOTE C, et al. A The evidence of benefits of exercise training in interstitial lung disease: a randomized controlled trial. Thorax 2017; 72: 610-9.

54.- LANKEN PN, TERRY PB, DELISSER HM, FAHY BF, HANSEN-FLASCHEN J, HEFFNER JE, et al. An official American Thoracic Society clinical policy statement: palliative care for patients with respiratory diseases and critical illnesses. Am J Respir Crit Care Med 2008; 177: 912-27.

55.- https://www.economist.com/graphic-detail/2017/04/27/ have-you-thought-about-your-final-wishes.

56.- RAJALA K, LEHTO JT, SAARINEN M, SUTINEN E, SAARTO T, MYLLÄRNIEMI M. End-of-life care of patients with idiopathic pulmonary fibrosis. BMC Palliat Care 2016; 15: 85.

57.- RUSSELL A-M, RIPAMONTI E, VANCHERI C. Qualitative European survey of patients with idiopathic pulmonary fibrosis: patients' perspectives of the disease and treatment. BMC Pulm Med 2016; 16: 10.

Correspondencia a:

Dr. José Luis Velásquez M.

Email: joseluisvelasquez992@gmail.com 